\title{
Évaluation du potentiel récréatif des environs d'une ville de la Grande Plaine hongroise : Debrecen
}

Evaluation of the recreational potential of the environs of a city of the Great Hungarian Plain : Debrecen

Bewertung des Erholungspotentials in der Umgebung einer Stadt der

Ungarischen Tiefebene : Debrecen

József Szabó, Timea Kiss et József Lóki

Traducteur : Simon Edelblutte

\section{OpenEdition}

\section{Journals}

Édition électronique

URL : http://journals.openedition.org/rge/2483

DOI : $10.4000 /$ rge.2483

ISSN : 2108-6478

Éditeur

Association des géographes de l'Est

Édition imprimée

Date de publication : 1 janvier 2003

ISSN : 0035-3213

Référence électronique

József Szabó, Timea Kiss et József Lóki, «Evaluationdupotentiel récréatif des environs d'une ville de la Grande Plaine hongroise : Debrecen », Revue Géographique de l'Est [En ligne], vol. 43 / 1-2 | 2003, mis en ligne le 08 décembre 2010, consulté le 07 septembre 2020. URL : http://journals.openedition.org/rge/2483 ; DOI : https://doi.org/10.4000/rge.2483

Ce document a été généré automatiquement le 7 septembre 2020

Tous droits réservés 


\section{Évaluation du potentiel récréatif des environs d'une ville de la Grande Plaine hongroise : Debrecen}

Evaluation of the recreational potential of the environs of a city of the Great Hungarian Plain : Debrecen

Bewertung des Erholungspotentials in der Umgebung einer Stadt der

Ungarischen Tiefebene : Debrecen

József Szabó, Timea Kiss et József Lóki

Traduction : Simon Edelblutte

\section{NOTE DE L'ÉDITEUR}

Cet article a été traduit de l'anglais, adapté et mis en forme par Simon Edelblutte (CERPA / Université Nancy 2).

\section{Introduction}

$1 \quad$ Les spécialistes de l'histoire du peuplement de la ville de Debrecen se réfèrent souvent au célèbre voyageur anglais, Robert Townson, qui, lors d'un de ses passages en Hongrie, a visité la ville en 1793. La présence d'une cité importante en cet endroit l'avait surpris, ainsi qu'il l'écrivait dans son journal : «À quelles circonstances Debretzin doit son existence, je ne le sais pas; je ne peux imaginer ce qui a pu pousser 30000 personnes à choisir pour la résidence un site sans source, matériau de construction, énergie et vin de qualité »(Townson, 1797). Cette remarque n'est cependant pas absolument exacte. Il semble en effet avoir échappé à l'attention de Townson qu'au voisinage de cette ville implantée au contact de deux régions naturelles d'altitude et de taille comparables mais néanmoins très différentes (Nyírség et Hajdúhát), s'étendent de vastes forêts, donc de l'énergie et 
des matériaux de construction, même si les maisons de bois n'ont jamais été caractéristiques de Debrecen. Par contre, la mention de l'absence de l'eau, facteur naturel pourtant essentiel dans le développement de la ville la plus importante, autrefois comme aujourd'hui, de la Grande Plaine hongroise, était pertinente. Le problème de l'alimentation en eau est resté d'actualité durant les deux derniers siècles et s'est même aggravé lors des dernières décennies.

Cet article s'attache essentiellement à l'un des aspects du déficit quantitatif et qualitatif en eau, lié au rôle de celle-ci dans la satisfaction de la demande en loisirs des populations des grandes villes modernes. Ce déficit, qui n'existait même pas au moment de la visite de Townson à Debrecen, est devenu dramatiquement d'actualité dans la deuxième moitié du XXe siècle. Dans le cas de Debrecen, cette question des loisirs est d'ailleurs liée à la forêt, mentionnée également par Townson en 1793. Le travail débute donc par une analyse de la situation hydrogéologique de Debrecen face à la demande en loisirs, puis montre comment cette situation initiale a été influencée par les formes de développement spatial de la ville, en insistant sur le rôle joué par des forêts voisines constamment modifiées par les impacts communautaires.

\section{Le cadre physique face au développement urbain et a la demande en loisirs}

3 Cette partie présente le cadre naturel de la ville de Debrecen et la demande grandissante en espaces de loisirs d'une ville en forte croissance démographique.

\section{A. Les grands traits géomorphologiques et biogéographiques}

4 La situation hydrogéologique de Debrecen (fig. 1) est principalement déterminée par deux facteurs :

5 - La ville est située dans la partie nord-est de la Grande Plaine hongroise (Alföld), à une altitude relativement importante par rapport au reste du pays. La région de Debrecen s'étend sur une partie d'un cône alluvial de plus de $5000 \mathrm{~km}^{2}$, construit par la Tisza et ses affluents au Pleistocène. Depuis la formation de la zone centrale du cône (Nyírség et, en partie, Hajdúhát) à la fin du Pléistocène et celle de dépressions plus récentes à ses marges, les rivières principales ont abandonné ce secteur qui est aujourd'hui un peu comme une "île" surélevée de 30 à $60 \mathrm{~m}$ en moyenne (avec un maximum de $80 \mathrm{~m}$ ) par rapport aux plaines environnantes, dont l'altitude est d'une centaine de mètres. Ainsi, les précipitations moyennes annuelles de cette partie surélevée de la Grande Plaine hongroise ne dépassent pas les 550 à $600 \mathrm{~mm}$ par an. L'écoulement s'y effectue, autour de la ville, sous la forme de courtes rivières de direction principale sud-sud-ouest, vers les parties les plus basses de la Grande Plaine ;

6 - Les dépôts fluviaux de la partie centrale du cône (Nyírség) ont évolué dès le Pléistocène en sables éoliens, déterminant la physionomie dunaire de la partie orientale de la région de Debrecen (Borsy, 1961). Par contre, dans la partie ouest - partiellement incluse dans le territoire municipal -, prennent place, intercalés dans les alluvions sableuses, des formations plus fines, vaseuses, parfois argileuses, mais surtout lœssiques. La surface de cette zone est formée d'une couche de lœss d'une épaisseur pouvant atteindre les $10 \mathrm{~m}$ (Szabó, 1965). Le comportement de ces terrains 
lœssiques peu disséqués (Hajdúhát) est, du point de vue de l'alimentation en eau et du potentiel agraire, différent de celui des sables éoliens du Nyírség; il en résulte donc des utilisations différentes. Sur les excellents tchernozioms du Hajdúhát, l'agriculture a prospéré depuis des siècles et occupe $90 \%$ de la superficie du secteur, alors que sur les marges orientales de la ville, la faible qualité des sols sableux n'a pas autorisé le développement d'une agriculture de haut niveau. Bien qu'il n'y ait pas de différence significative dans le total moyen annuel des précipitations entre les deux secteurs - seule une légère augmentation est sensible en s'avançant de l'Hortobágy ${ }^{1}$ vers les marges orientales de la Grande Plaine - le taux de boisement dans la région des sables éoliens est, même encore aujourd'hui avec $30 \%$, considérable, se maintenant largement au-dessus de celui du reste de la Grande Plaine, inférieur à $10 \%$. Les forêts, dans leur majorité, ne sont pas primaires et sont issues de reboisements effectués après d'anciens défrichements. Le peuplement a aussi évolué, les chênes originels ayant été remplacés par des robiniers et des plantations de pins. Néanmoins, grâce à ces forêts, le paysage des confins orientaux (Nyírség) de la ville est plus varié et attractif que celui de la partie occidentale (Hajdúhát). La forêt s'étend principalement sur les dunes de sables; entre elles, essentiellement dans les petites vallées peu profondes d'orientation sud-ouest évoquées plus haut, dominent d'importantes surfaces de prés et de prairies.

Figure 1 : Carte de localisation des environs de Debrecen

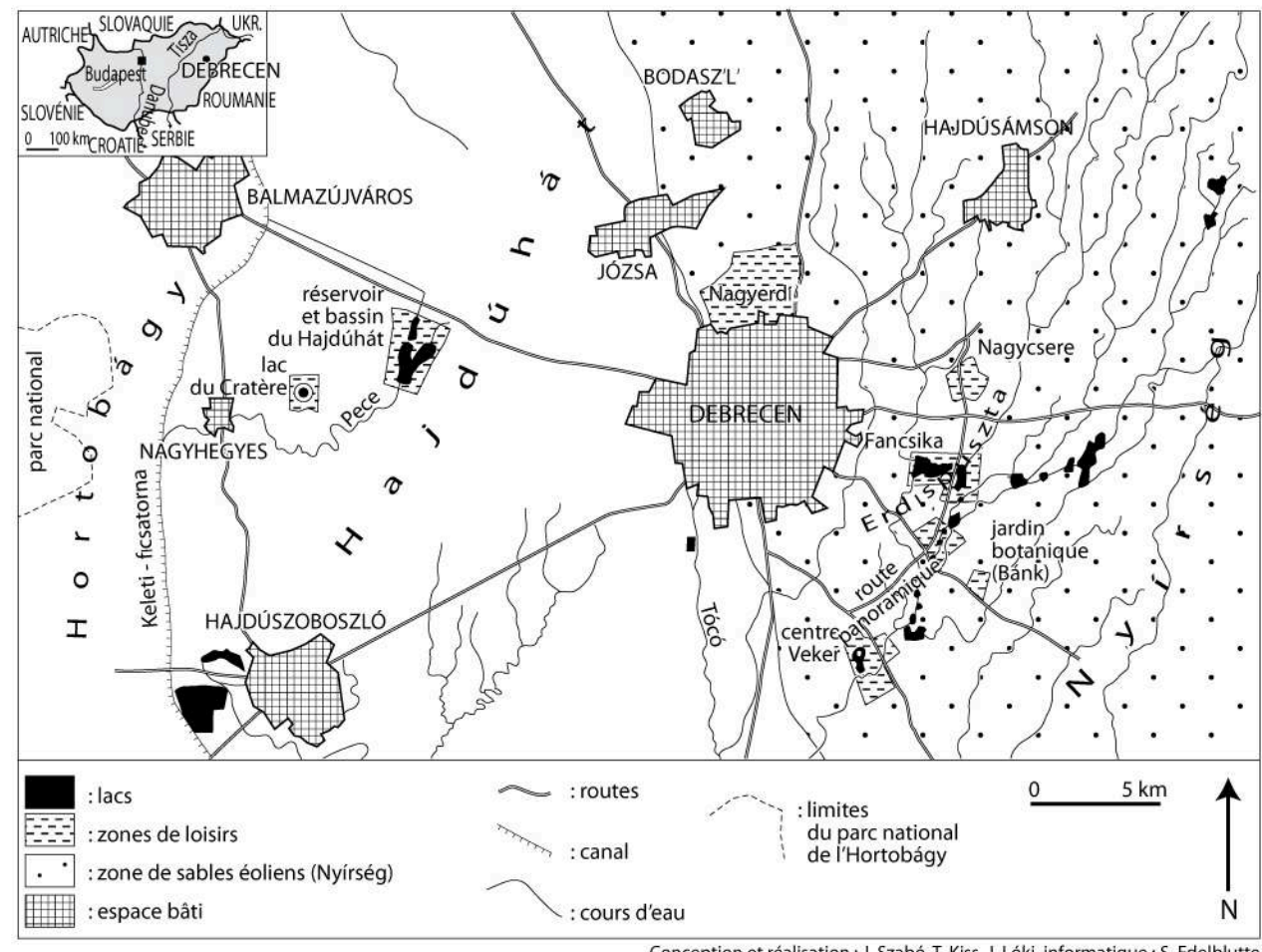

7 Aux limites nord de la ville, un vaste et homogène morceau de la forêt originelle a été préservé depuis la période protohistorique. C'est la fameuse Grande Forêt de Debrecen (Nagyerdı), qui, au moment de la visite de Townson, accueillait les premières activités de loisirs des habitants de Debrecen. Ainsi, le temps d'un week-end, ou pour une sortie occasionnelle, les marges sud de la Grande Forêt, avec leur tapis de muguet sous couvert de chênes, étaient un but apprécié de promenade. 


\section{B. Croissance urbaine, demande et potentiel de loisirs}

Debrecen, qui comptait 30000 habitants au temps de Townson, a atteint 100000 habitants au moment de la Première Guerre Mondiale. La demande en eau croissante de cette ville était alors satisfaite par la nappe phréatique du cône alluvial, captée sous la Grande Forêt. Avec une consommation quotidienne atteignant $60000 \mathrm{~m}^{3}$ au début des années 1970, et alors que les sources ne pouvaient en satisfaire que la moitié (TIVIZIG Tiszántúli VíZügyi IGazgatóság, 1974), la nappe phréatique commença à s'épuiser (fig. 2), entraînant une dégradation des peuplements forestiers. Cela a mis en lumière la nécessité pour la ville de trouver de nouvelles sources d'approvisionnement en eau. Ainsi, en 1974, la construction d'une station élévatoire et d'un système d'aqueducs a-telle débuté. Cet ensemble, dont la capacité est constamment améliorée, amène à la ville l'eau de la Tisza via un prélèvement dans le Grand Canal de l'Est (Keleti FI csatorna), à $20 \mathrm{~km}$ de Debrecen. Après avoir relevé l'eau d'environ $30 \mathrm{~m}$, le système de conduites traverse la région de lœss du Hajdúhát, où une partie de l'eau est prélevée pour l'irrigation. Grâce à ce système, la ville a résolu son problème d'alimentation en eau, mais n'a pas réussi à répondre à la demande en eau pour les loisirs d'une population qui a atteint entre-temps plus de 200000 habitants. Ainsi, même la demande en eau des thermes, qui jouissent pourtant d'une réputation nationale depuis les années 1920, ne pouvait plus être satisfaite ${ }^{2}$.

Figure 2 : Prélèvements et niveau de la nappe à Debrecen entre 1963 et 1994

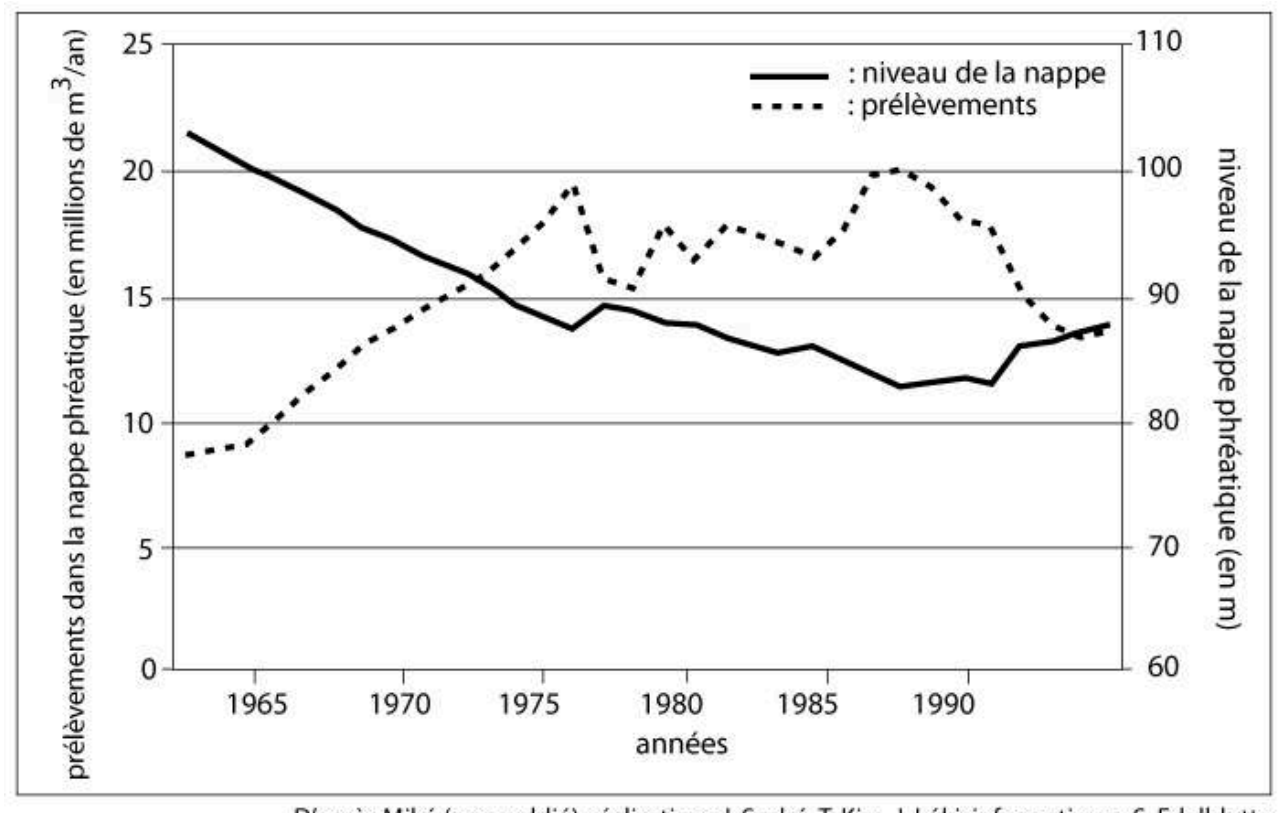

D’après Mikó (non publié), réalisation : J. Szabó, T. Kiss, J. Lóki, informatique : S. Edelblutte

Parallèlement, la demande en loisirs a cru encore plus vite que le nombre d'habitants, grossi par l'arrivée massive de ruraux des régions environnantes, principalement installés dans d'énormes collectifs de dix étages. Les structures récréatives anciennes étaient évidemment insuffisantes pour satisfaire ce besoin croissant, particulièrement dans le domaine des activités nautiques et des loisirs verts, et ce même au prix d'une destruction possible de la Grande Forêt. Ce risque a été un des stimulants essentiels d'un projet à grande échelle, qui désirait résoudre ces problèmes vitaux par l'usage 
des ressources naturelles en eau des environs pour créer de nouveaux espaces aquatiques, en étendant ou en transposant partiellement les zones de loisirs déjà existantes (Papp, 1974). Les zones boisées (Erdıspuszta) des confins orientaux de la ville, disséquées par les vallées peu profondes des petits cours d'eau, furent alors considérées comme de nouvelles zones de loisirs. Il y eut également des velléités d'utiliser une partie de l'eau de la Tisza transportée vers la ville pour former une zone de loisirs dans la région de lœss (Hajdúhát), pourtant moins attractive. Comme nous le verrons dans les parties suivantes, ces expériences prometteuses ne pourront pas satisfaire la demande urbaine en espaces de loisirs nautiques, d'autant plus qu'à cause du manque de moyens financiers, certains équipements difficilement achevés et à l'impact hydrogéologique considérable, montrent des signes de dégradation et pourraient être perdus sans investissements supplémentaires.

\section{La Grande Forêt (Nagyerdo), espace de loisirs traditionnel de Debrecen} de forme circulaire atteignant presque $11 \mathrm{~km}^{2}$ et bordant la ville au nord.

Figure 3 : L'occupation du sol au cœur de la Grande Forêt (Nagyerdo)

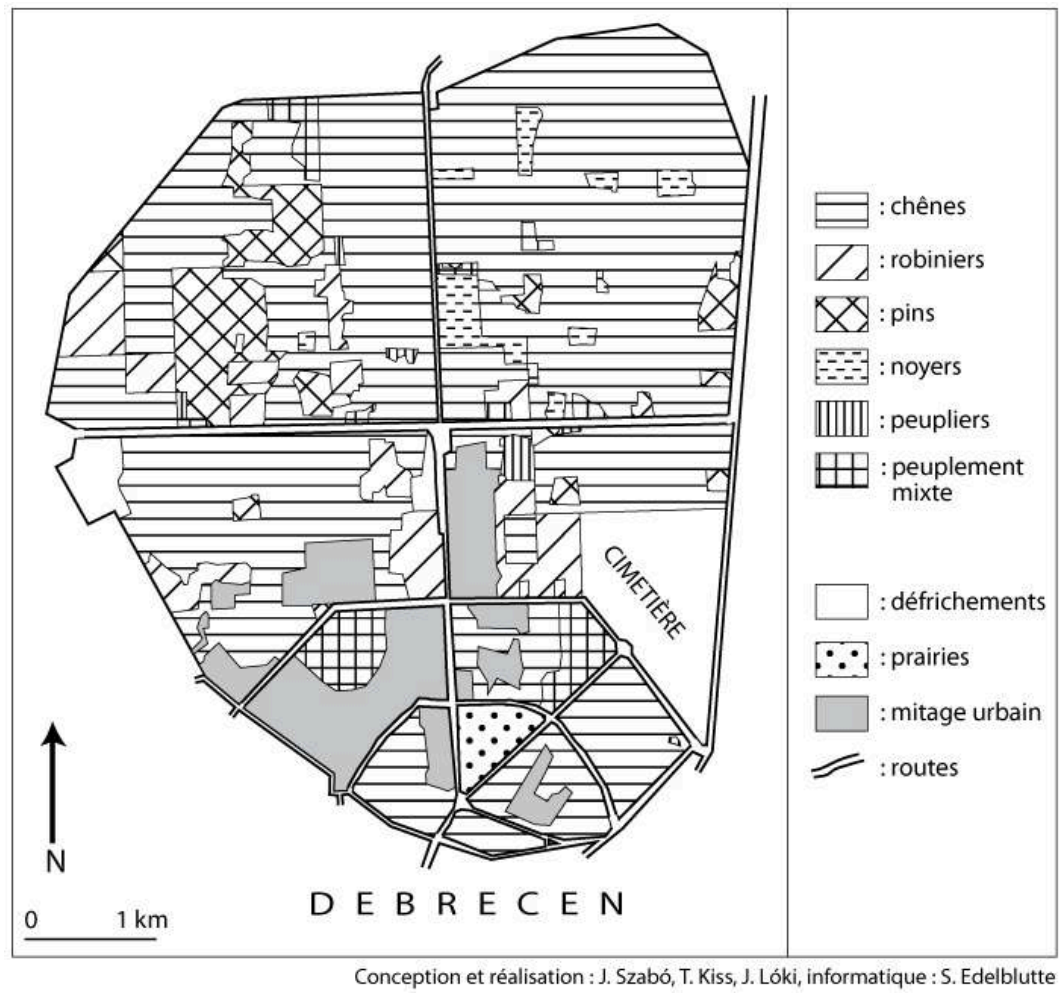

\section{A. Un milieu original utilisé depuis longtemps pour les loisirs}

11 Cette forêt, qui a aujourd'hui perdu beaucoup de son côté " naturel », peut néanmoins être perçue comme une forêt primaire, dans le sens que le secteur a toujours été couvert par une forêt plus ou moins continue, après les fluctuations climatiques du début 
de l'Holocène. Plus tard, il est resté forestier durant la phase Atlantique et aussi au début de la période historique. En dépit d'une pression constante, cette forêt a maintenu jusqu'à nos jours, dans des secteurs réduits toutefois, son peuplement caractéristique associant le chêne (Convallario-Quercetum roboris) et un tapis de muguet au sol (Jakucs, 1989). Ce type de forêt était commun à tous les espaces sableux du Nyírség et la Grande Forêt en est donc l'une des dernières reliques, même si ce n'est pas la plus vaste. Dans son état originel, l'une de ses caractéristiques était la formation, due au haut niveau de la nappe phréatique maintenu par la présence de la forêt, de petits marais dans les creux entre les dunes de sable. Ce type d'endroit est encore mentionné au XIX ${ }^{\mathrm{e}}$ siècle.

Les transformations à grande échelle de la Grande Forêt ont commencé à la fin du XVIII siècle, quand l'ensemble des fossés entourant la ville ont été comblés et que l'aménagement paysager des parties de forêt proches de la ville a été pour la première fois planifié (Kerényi, 1997). L'utilisation de ces espaces dans un but de loisirs remonte ainsi au début du XIX ${ }^{e}$ siècle. Commencé aux marges sud de la forêt, le plan a été mis en œuvre en trois étapes et la superficie initialement protégée a été considérablement transformée.

- Durant la première phase, dans la première moitié du XIX ${ }^{\mathrm{e}}$ siècle, la construction du centre de loisirs de Vigadó et de sa piscine a simplement réduit la surface de la Grande Forêt et ne l'a pas fondamentalement bouleversée, tout comme la décision du Conseil Municipal d'y installer un camp de bûcherons.

- La deuxième phase, peut-être la plus significative en matière de changement, s'est produite durant l'Entre-deux-guerres, quand l'extension de l'habitat a provoqué un déclin considérable de la surface boisée.

- Enfin, la troisième phase a commencé au début des années 1950 et s'est poursuivie jusqu'à nos jours, avec l'apparition d'usines, blocs de béton tranchant dans le paysage et nuisibles au potentiel de loisirs de la Grande Forêt.

\section{B. Des problèmes variés affectent la Grande Forêt}

En plus de la réduction de la surface boisée, la baisse du niveau de la nappe phréatique due au drainage, la proportion déclinante de jeunes plants de chêne issus de repousse naturelle, les défrichements et les plantations de robiniers ont été les plus importants facteurs aggravants de problèmes qui perdurent jusqu'aujourd'hui. Un des éléments les plus pénalisants de la réduction de la surface boisée en vue d'un développement ultérieur par les loisirs, a été la parcellisation de la forêt, liée à la construction de lotissements épars, de puits pour les travaux d'irrigation, d'usines, ou tout simplement à la réalisation de clairières. Ce mitage s'observe très bien sur les photographies aériennes de cette partie sud de la Grande Forêt et est représenté sur la figure 3. À cause de ces défrichements, le climat de la région toute entière a évolué, devenant plus chaud et plus sec. Ces changements climatiques ont affecté les bois encore intacts, dégradant les conditions de développement des plants les plus jeunes. Ainsi, les effets négatifs de la multiplication et de la dispersion des défrichements se sont accumulés et étendus à l'ensemble de la forêt.

Un autre problème, presque aussi grave que la réduction de la surface boisée, a été la perte du caractère uniforme de l'association chêne-muguet originelle. En effet, et à part les 31 ha protégés depuis 1939, seule une petite surface de 7 ha a conservé cette 
association dans un bon état relatif. De plus, les plants de seconde génération, repousse naturelle dans les zones où les arbres ont été abattus, ont été de moins en moins nombreux et sont aujourd'hui présents, pour les plus anciens - âgés d'une centaine d'années - sur seulement $5 \%$ de la superficie boisée totale. Les semis de chênes sont ainsi aujourd'hui dominants, et l'âge des trois-quarts des plants ne dépasse pas les 70 ans. Au XIXe siècle, au plus fort des défrichements de la Grande Forêt, la proportion des chênes pédonculés originels (Quercus robur) a progressivement diminué au profit des plants de robiniers (Robinia pseudo-acacia), pins et autres espèces (fig. 3). Certes, d'un point de vue esthétique, beaucoup d'éléments subjectifs entrent en compte dans l'évaluation de la forêt. Cependant, il ne fait aucun doute que les traces de l'association chêne-muguet non modifiée - qui ne peut être que le résultat de siècles d'évolution est plus intéressante que les zones modifiées dans un but pratique ou productiviste.

Depuis des siècles, la Grande Forêt a été considérée comme le poumon de la ville, la rafraîchissant en été grâce à la diffusion de son air plus clair et relativement plus humide. Aujourd'hui, une telle fonction serait encore nécessaire, mais elle est non seulement contrariée par les modifications évoquées plus haut, mais aussi menacée par la croissance constante de la pollution de l'air urbain et de son impact sur la vie de la forêt. Tout autant que le trafic automobile qui pénètre de plus en plus profondément dans la forêt, la traversant et l'enserrant de routes de plus en plus nombreuses, l'augmentation programmée du nombre de bâtiments à construire dans la forêt peut être justement dénoncée.

La Grande Forêt, de plus en plus petite et détériorée, est de nos jours de moins en moins capable de servir d'espace de loisirs pour la ville. Cependant, ce qui reste de son potentiel récréatif pourrait être mieux mis en valeur si une partie de la fonction de loisirs était transférée dans d'autres endroits adéquats aux alentours de Debrecen.

\section{L'Erdospuszta, nouvel espace de loisirs de la ville}

17 L'Erdospuszta, en hongrois « prairie arborée », est la partie méridionale du Nyírség, à l'est-sud-est de Debrecen (fig. 1 et fig. 4). C'est plus une région botanique que géographique. Sa superficie est d'environ $130 \mathrm{~km}^{2}$ et elle est pour l'essentiel constituée de sables éoliens, disposés au Pléistocène sous la forme de dunes paraboliques aux côtés ouest tronqués. Les surfaces les plus basses correspondent à d'anciennes vallées de direction nord-est/sud-ouest. 
Figure 4 : Peuplements forestiers et loisirs dans l'Erdospuszta

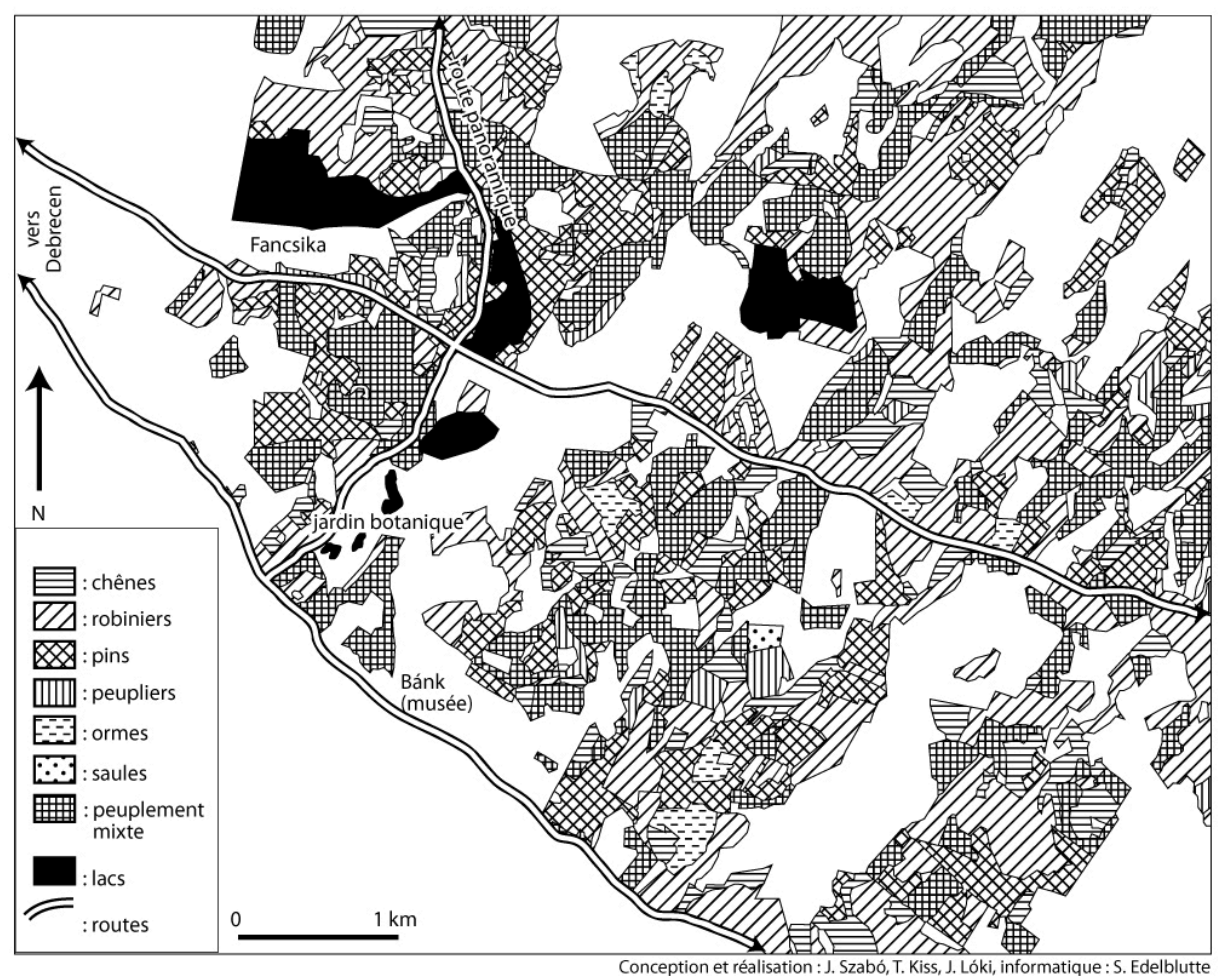

\section{A. D'une terre agricole à un espace consacré aux loisirs}

18 Au Moyen-Âge, de gros villages prospéraient dans ce pays de forêt, de marécages et de dunes; les habitants y pratiquaient une économie agro-pastorale. Cependant, entre le $\mathrm{XVI}^{\mathrm{e}}$ et le XVIII ${ }^{\mathrm{e}}$ siècle, le secteur a perdu beaucoup de sa population et la ville de Debrecen a pu ainsi acquérir l'essentiel des forêts, qui sont progressivement devenues prédominantes dans ces zones autrefois partagées entre des prairies sur sable, des prés et des marécages. Le paysage a de nouveau commencé à changer au début du $\mathrm{XIX}^{\mathrm{e}}$ siècle, lorsqu'à la suite de l'installation de nouvelles populations, les défrichements ont débuté ; la forêt a ainsi été réduite de moitié, remplacée par des terres cultivées et des prés. Le paysage a été également modifié par le drainage des zones humides et des marécages. Commencés à la fin du XIX ${ }^{e}$ siècle (1892), ces travaux ont provoqué l'affaissement considérable du niveau de la nappe phréatique et donc l'assèchement et la disparition des chênaies originelles et le déclin des chênaies subsistantes. Afin de combler ce déficit en eau en stoppant la baisse du niveau de la nappe phréatique, un nouveau système de canaux a donc été mis en place. Ces travaux ont été entrepris simultanément à la transformation de certains secteurs de l'Erdospuszta en espaces de loisirs (Papp, 1974). Cette région était en effet, dans les environs de Debrecen, le meilleur endroit pour les loisirs, et environ $46 \mathrm{~km}^{2}$ ont ainsi été aménagés dans ce but (fig. 1 et fig. 4). La partie principale du plan de développement consistait en la mise en place de 14 lacs (d'une superficie de 3000 ha pour une profondeur comprise entre 1 et $1,4 \mathrm{~m})$ par le barrage des petits cours d'eau s'écoulant dans les vallées parallèles nord-est/sud-ouest. Dans les environs d'une ville manquant d'eau comme Debrecen, ces lacs constituent un atout majeur pour les activités récréatives. Certains d'entre eux sont néanmoins réservés à l'irrigation. 
La transformation de l'Erdospuszta en espaces de loisirs avait été guidée par plusieurs raisons :

- les zones de loisirs sont aisément accessibles par les transports en commun, la voiture ou le vélo, car ils sont situés le long d'une radiale quittant la ville et à proximité de la bien nommée « route panoramique » qui contourne la partie sud de la ville en recoupant es radiales;

- l'Erdospuszta offre, dans les environs de la ville, les paysages les plus variés et les panoramas les plus riches. En effet, les dénivellations, certes modérées (entre 13 et $15 \mathrm{~m}$ pour un cercle de $100 \mathrm{~m}$ de rayon), sont cependant importantes par rapport au reste de la Grande Plaine ;

- la proportion de surface boisée, d'une valeur moyenne oscillant entre 40 et $50 \%$, est la plus importante de la Grande Plaine ;

- les prairies steppiques sèches des versants des dunes, les zones humides de fonds de vallées et les marécages abritent une faune et une flore diversifiées. En raison de leur abandon depuis des siècles, ces zones, protégées de nos jours tout en étant ouvertes au public, ont servi de refuge pour des espèces relictuelles et menacées, (Gyarmaty et al., 1966);

- l'usage le plus adapté de ces sols sableux, peu fertiles et donc médiocres pour l'agriculture, est finalement de les laisser en forêt ou de les aménager pour le tourisme et/ou les loisirs.

Lors de la mise au point du projet de transformer l'Erdospuszta en espace de loisirs, la possibilité d'une fréquentation très importante le week-end a été envisagée. Ainsi, pour en éviter les inconvénients, les concepteurs ont-ils créé quatre centres de loisirs (fig. 1 et fig. 4), dispersés dans toute la zone et répondant à demandes différentes (Módi et al., 1989) :

- la forêt de Nagycsere est consacrée au divertissement en extérieur (terrains de sports, aires de jeux...);

- les lacs de Fancsika (130 ha de surface en eau) sont utilisés par les pêcheurs ;

- le jardin botanique et le musée de Bánk abritent l'office du tourisme pour toute la zone et présentent sa flore et sa faune, ainsi que la vie traditionnelle des bûcherons, des fermiers et des éleveurs ;

- les loisirs sportifs sont à Veker, où les terrains de sport, les espaces de promenade et les campings ont été installés.

\section{B. Des résultats mitigés}

21 L'Erdospuszta est fréquentée par plusieurs milliers de personnes chaque année (le jardin botanique atteint les 20000 visiteurs annuels), mais cette fréquentation est inférieure de $25 \%$ à celle prévue (Sípos, 1980). Une des causes principales de ce résultat médiocre et l'amorce d'une dégradation du milieu, essentiellement due à la pénurie d'eau dans cette zone. Le total annuel moyen annuel des précipitations, sur une série de 50 ans, est de $550 \mathrm{~mm}$, mais durant les vingt dernières années, parallèlement à la mise en place des lacs, les précipitations se sont abaissées à $540 \mathrm{~mm} / \mathrm{an}$, desquels $500 \mathrm{~mm}$ sont perdus par évaporation (Lovas, Hajek, 1989). Le ruissellement, très faible, est donc insuffisant, surtout en saison sèche, pour assurer l'alimentation des lacs artificiels. Par conséquent, ces plans d'eau, pourtant éléments essentiels de l'attractivité du secteur, ont commencé à se dégrader. Des mesures effectuées par nos soins ont montré que les petits cours d'eau alimentant ces lacs y apportent des eaux de moyenne ou mauvaise qualité, ce qui provoque, en été, une importante eutrophisation. L'eau, peu 
profonde, se réchauffe alors et est colonisée par des algues et des plantes aquatiques. Ce phénomène, rapide, a commencé aussitôt la mise en place des lacs. Par ailleurs, et alors que leur profondeur initiale était faible, ils ont commencé à se remplir très tôt en raison de l'important apport d'éléments organiques et de sédiments très fins transportés par les cours d'eau. Nous avons essayé de déterminer la vitesse de remplissage lors de deux campagnes de mesures entre 1995 et 1997. Nous avons ainsi établi que le comblement est principalement le fait d'éléments organiques. La vitesse annuelle de remplissage était de $1 \mathrm{~cm} /$ an entre 1976 et 1990, avant de s'élever à 3,1 cm/an à partir de cette dernière année. Cela implique que l'épaisseur des sédiments accumulés durant 20 ans atteint $40 \mathrm{~cm}$ dans ces lacs peu profonds - au maximum 1,4 m. Si cette tendance n'est pas stoppée, l'usage récréatif de ces lacs pourrait ne plus être possible dans une décennie ou deux, lorsqu'ils seront entièrement remplis. Leur déclin déjà avancé est prouvé par la diminution de leur fréquentation par les pêcheurs, évidemment liée à la faiblesse grandissante du peuplement piscicole. Lors d'années très sèches, comme en 1995, les lacs peuvent même être totalement asséchés. Seuls des investissements supplémentaires pourraient résoudre ce problème, voire améliorer la situation, en autorisant de fréquents dragages et surtout la mise en place d'une alimentation en eau extérieure, d'ailleurs prévue dans les projets initiaux.

La faiblesse grandissante des précipitations représente également une grande menace pour les peuplements forestiers. La surface couverte de chênes pédonculés a été réduite par les défrichements précoces de $100 \%$ de la zone à $17 \%$ seulement (Sípos, 1980) et elle continue à décroître à cause de l'abaissement de la nappe phréatique. Ce processus n'a même pas été entièrement stoppé par les travaux d'aménagements hydrauliques jusqu'ici mis en œuvre.

Un autre problème important est la méconnaissance, par le grand public, de l'Erdospuszta en tant qu'espace de loisirs, ce qui ne favorise évidemment pas sa fré quentation. Un nombre plus important d'usagers pourrait en effet procurer les moyens financiers pour l'entretien et le développement des infrastructures de loisirs, le dragage régulier des lacs et la mise en place d'une alimentation en eau extérieure, à partir du Grand Canal de l'Est et via la Grande Forêt qui pourrait donc également profiter de cette eau.

\section{Le rôle récréatif modeste du Hajdúhát}

Le Hajdúhát commence pratiquement en bordure ouest de la ville. Sa limite est soulignée par la large et plane vallée du Tócó (fig. 1), qui s'écoule du nord vers le sud.

\section{A. Une surface lœssique au faible potentiel récréatif}

Cette région de lœss s'étend sur environ $15 \mathrm{~km}$ d'est en ouest, avec une altitude moyenne de $120 \mathrm{~m}$. La surface lœssique est ici parfaitement plane et domine, d'environ $30 \mathrm{~m}$ et par un talus assez raide, l'Hortobágy, ancien fond de vallée de la Tisza. Au pied du talus, s'écoule le Grand Canal de l'Est (Keleti-FIcsatorna), qui apporte, pour l'irrigation, l'eau de la Tisza plus vers le sud. Le rôle géomorphologique du talus est important car il a entraîné une érosion régressive à partir de quelques vallées qui ont donc disséqué la lisse surface lœssique. Les cours d'eau de ces vallées traversant la région de lœss vers le sud ou l'ouest, ont un débit peu important et sont parfois à sec en 
été. La capacité hydrique de la région lœessique est, de plus, faible, en raison de sa structure géologique, décrite plus haut. Cependant le lœss vaseux, et parfois argileux à l'ouest de Debrecen, la proportion de lœss et d'argile est généralement plus haute que celle de sable - et les fréquentes zones de tourbe rendent les vallées, en théorie, capables de retenir l'eau. Avec une profondeur qui excède, en certains endroits, $10 \mathrm{~m}$, ces larges vallées pourraient supporter la mise en place de réservoirs considérables. Ces conditions favorables ont été prises en compte dans le programme "Système Hydraulique à Multiples Objectifs " (TIVIZIG, 1974). L'idée était de transférer, par pompage dans le Grand Canal de l'Est, $3,5 \mathrm{~m}^{3} / \mathrm{s}$ d'eau pour créer des réservoirs à multiples objectifs (irrigation, loisirs...). Le plan prévoyait qu'une petite partie de cette eau serait transférée jusqu'à l'Erdospuszta via la Grande Forêt. Dix réservoirs devaient être construits à l'ouest de Debrecen, sur une surface de 741 ha et pour une capacité utile de 13,4 millions de $\mathrm{m}^{3}$ réservés principalement à un usage agricole. Cependant, pour des raisons financières, le projet ne s'est pas concrétisé et un seul de ces réservoirs ( 60 ha pour 1,7 million de $\mathrm{m}^{3}$ ) a finalement été construit sur le cours amont du Pece. Grâce à cet aménagement, 1170 hectares ont été irrigués, mais à cause du coût élevé de cette eau pompée depuis le Grand Canal de l'Est, la taille de la surface irriguée n'a pas progressé sensiblement. Parallèlement, une utilisation secondaire pour les loisirs est spontanément apparue (pêche, sports nautiques, naturisme...). Cependant et malgré des conditions initiales favorables, l'absence d'infrastructures appropriées limite fortement la fréquentation. Ainsi, le manque de fonds ayant rendu impossible la mise en place des autres lacs et la demande urbaine en eau n'ayant pas crû comme prévu à cause de l'explosion du prix de l'eau, la capacité de pompage dans le canal est aujourd'hui largement sous exploitée.

Un petit bassin nautique de 2 ha et d'une capacité de $22000 \mathrm{~m}^{3}$ a malgré tout été installé dans la vallée du Pece à l'amont du réservoir. C'est aujourd'hui le seul bassin de baignade en eau naturelle dans un rayon de $13 \mathrm{~km}$ autour de la ville. Bien que son environnement, fait de champs de maïs, ne soit pas très enthousiasmant, sa popularité a grandi très vite dans les années 1980 et il était alors fréquenté par plus de 50000 personnes par an. Cette fréquentation est aujourd'hui en baisse, à cause de la température faible et variable de l'eau amenée ici par des conduites et en raison de l'augmentation des coûts de transport.

\section{B. Le lac du Cratère}

Dans cette région du Hajdúhát au relief monotone, l'étang anthropique apparu en 1961 et appelé lac du Cratère (fig. 1 et fig. 5), est un endroit original et prometteur dans l'optique des loisirs. Il est issu de la plus forte explosion de gaz de l'histoire de la recherche d'hydrocarbures en Hongrie. L'accident a produit un cratère circulaire de $200 \mathrm{~m}$ de diamètre au cœur de la plaine. Il est entouré d'un bourrelet de terre dominant de 6 à $8 \mathrm{~m}$ la surface de la plaine environnante et cachant un bassin de $7100 \mathrm{~m}^{3}$ qui atteint encore aujourd'hui presque $10 \mathrm{~m}$ de profondeur. Le niveau de l'eau, maintenu par des sources souterraines, se situe à $4 \mathrm{~m}$ plus bas que les environs. Le lac est donc entouré d'une rive très raide, aujourd'hui recouverte d'une végétation dense et offrant un panorama étonnant. Cependant, et bien qu'il soit accessible par une route pavée, il n'est fréquenté que par un petit nombre de pêcheurs. Sa taille réduite est certes incompatible avec une fréquentation importante, mais le fait qu'il tranche totalement dans un paysage dépourvu de relief est surprenant et pourrait peut-être être exploité. 
Figure 5 : Le lac du Cratère de Nagyhegyes.

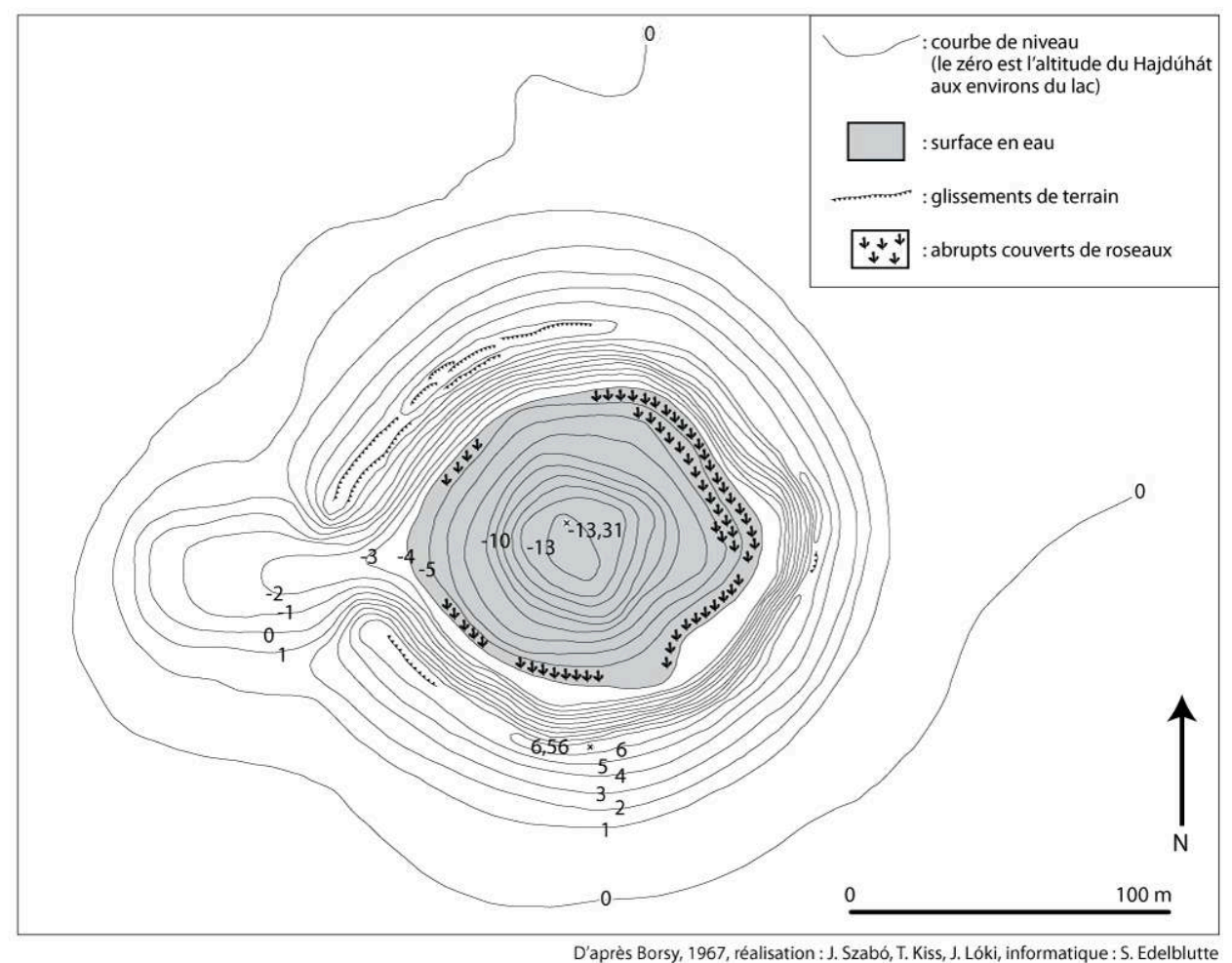

En résumé, le Hajdúhát ne joue qu'un rôle modeste dans la satisfaction de la demande en loisirs des habitants de Debrecen. En raison de ses maigres atouts naturels, aucun changement décisif ne devrait y avoir lieu; néanmoins, la proximité de la principale ressource en eau du secteur, le Grand Canal de l'Est et donc la mise en eau possible d'autres lacs, pourrait, par extension, soulager la Grande Forêt et l'Erdospuszta en répartissant mieux la fréquentation récréative.

\section{Conclusion}

En vue de l'entrée à brève échéance de la Hongrie dans l'Union européenne, cette étude montre le demande croissante en loisirs d'une population urbaine longtemps mal pourvue dans ce domaine, tant pour des raisons naturelles liées à l'aridité du milieu des confins orientaux de la Grande Plaine hongroise que pour des raisons politiques.

Malgré ces conditions difficiles et à la lumière des faits évoqués dans le développement, il peut cependant être établi que les environs de Debrecen sont capables de satisfaire la demande croissante des habitants de la ville en matière de loisirs, mais cela seulement si une alimentation en eau suffisante et maintenue. À ce prix seulement, la dégradation croissante de la Grande Forêt pourra être enrayée, les lacs de l'Erdospuszta correctement alimentés et le système d'irrigation du Hajdúhát complété par des aménagements récréatifs. Enfin, il ne faut pas oublier non plus qu'avant la réalisation de ces projets, la protection et le simple maintien des zones de loisirs actuelles, par l'amélioration des infrastructures et une meilleure promotion du site, doit d'abord être assuré. 


\section{BIBLIOGRAPHIE}

Borsy Z. (1961). - A Nyírség természeti földrajza (Géographie physique du Nyírség), Budapest, 167 p.

Borsy Z. (1967). - The crater of Nagyhegyes (Le cratère de Nagyhegyes), Acta Geographica Debrecina, 1966/67, Tomus XII-XIII, p. 84-100.

Gyarmaty I., Buka L., Papp L., Rácz Y. (1966). - A dél-nyírségí Erdıspuszták (La région de l'Erdıspuszta au sud du Nyírség), Tájak-Korok-Múzeumok Kiskönyvtára, n 547.

Jakucs P. (1989). - A Nagyerdı vegetációja (La couverture végétale de la Grande Forêt), Calandrella, p. 6-17.

Kerényi A., éd. (1997). - A debreceni Nagyerdei Park (Le Parc de la Grande Forêt de Debrecen), 166 p. Lovas M., Hajek H. (1989). — Erdıspuszta. Bemutatóház és fivészkert (Le jardin botanique et le musée de l’Erdıspuszta), Tájak-Korok-Múzeumok Kiskönyvtára, nº 355.

Módy G., Lovas M., Puskás P. (1989). - Debrecen-Erdıspuszta (L'Erdıspuszta dans les environs de Debrecen), Tájak-Korok-Múzeumok Kiskınyvtára, n 354.

Papp F. (1974). - Debrecen térségének vizgazdálkodása (La gestion de l'eau dans la région de Debrecen), Vízügyi Közlemények, p. 485-510.

Sípos G. (1980). - Debrecen Erdıspusztai erdık ökológiai és ökonómiai optimalizálásának összefüggés vizsgálata (Évaluation de la région de l'Erdıspuszta pour une optimalisation écologique et économique), Thèse, p. 192.

Szabó J. (1965). - Geomorfológiai megfigyelések a Hajdúháton (Les comportements géomorphologiques du plateau de lœss de l'Hajdúhát), Acta Geographica Debrecina, 1964/65, p. 197-220.

TIVIZIG - Tiszántúli VÍZügyi IGazgatóság (Agence de l'eau du bassin de la Tisza) (1974). — A Hajdúsági Többcélú Vizgazdálkodási Rendszer (Le système hydraulique à multiples objectifs), Manuscrit, Debrecen, $30 \mathrm{p}$.

Townson R. (1797). - Travels in Hungary (Voyages en Hongrie), London, 538 p.

\section{NOTES}

1. L'Hortobágy correspond à la partie centre-nord de l'Alföld. Un Parc National, centré sur la zone la plus humide et inscrit sur la liste du Patrimoine Mondial de l'UNESCO depuis 1999, y a été créé.

2. La ville voisine d'Hajdúszoboszló (fig. 1) est aujourd'hui un centre thermal plus réputé que Debrecen. 


\section{RÉSUMÉS}

Avec le développement de la vie citadine moderne, les besoins récréatifs de la population augmentent à un rythme accéléré. Pour les satisfaire, l'environnement naturel joue un rôle croissant, justement à cause du caractère urbain du mode de vie. Les villes entourées de reliefs accidentés, de nombreux plans d'eau et d'une végétation abondante sont favorisées dans ce domaine. De ce point de vue, les villes de la Grande Plaine hongroise, au relief monotone et à l'aridité prononcée, ne sont pas dans une situation avantageuse. Debrecen, devenue la deuxième ville du pays au cours de la dernière décennie, dispose ainsi de possibilités plus restreintes que la moyenne.

L'étude recense les dispositions naturelles en matière de loisirs des environs de cette ville, située au contact de deux parties différentes de la plaine. Elle démontre d'abord la dualité géomorphologique des alentours de la ville (à l'est, un espace constitué de dunes de sables éoliens sous couvert forestier dominant - le Nyírség ; à l'ouest une dorsale de lœss - Hajdúhát), puis met l'accent sur les conséquences négatives de la pauvreté en eaux superficielles de la ville et de ses périphéries. Elle s'attache ensuite aux tentatives et projets de lutter contre l'aridité en introduisant l'eau du Grand Canal de l'Est sur des espaces au potentiel récréatif certain, avant d'analyser l'importance récréative et le dépérissement de la Grande Forêt du nord de la ville. Enfin, elle examine le déclin des étangs de loisirs artificiels de l'Erdıspuszta, crées dans les dunes du Nyírség, avant d'évoquer, dans la dernière partie, le modeste potentiel récréatif de la dorsale de lœss de l'ouest de la ville.

En conclusion, les auteurs soulignent que le manque d'eau nécessaire au développement des loisirs dans les environs de la ville pourrait être résolu en dérivant une partie de l'eau du Grand Canal de l'Est destinée actuellement à la ville seule.

With the spread of modern urban life, the recreational demands of the population are growing at an accelerating rate. In satisfying these demands, the natural environment assumes an everincreasing role, due to the character of urban life. Cities surrounded by hilly relief, abundant vegetation and rich natural waters are therefore advantaged. The cities of the Great Hungarian Plain, with its monotonous relief and pronounced aridity, are not favourably placed. The opportunities for Debrecen, which has grown into the second largest city in the country in the last decade, are even more restricted.

This study reviews the natural resources of this city, situated between two different parts of the plain, with respect to leisure activities. It describes the dual geomorphological character of the city's environs (to the east, an area of mostly forested sand dunes - Nyírség ; to the west a loess plain - Hajdúhát), laying emphasis on the negative consequences of a shortage of surface water in the city and its outskirts. It presents and evaluates efforts to counter the aridity by introducing the water of the East Main Canal into the areas with definite recreational potential, before analysing the recreational importance and decay of the Great Forest in the northern part of the city. Lastly, the study examines the decline of the artificial leisure lakes of the Erdıspuszta, created in the dunes of the Nyírség, before mentioning, in the last part, the modest recreational potential of the loess plain west of the city.

In conclusion, the authors emphasise that the lack of necessary water for the development of recreation in the environs of the city can be resolved by diverting part of the water of the East Main Canal presently destined for the city itself.

Mit der Entwicklung des modern städtischen Lebens vergrößern sich die Erholungsbedürfnisse der Bevölkerung in beschleunigtem Rhythmus. Um sie zu befriedigen, spielt die natürliche Umwelt eine zunehmende Rolle, gerade wegen der städtischen Lebensweise. Die Städte mit 
reliefreicher Umgebung, zahlreichen Wasserflächen und reicher Vegetation sind in dieser Hinsicht begünstigt. Diesbezüglich sind die Städte der Grossen Ebene Ungarns mit monotonem Relief und ausgeprägter Aridität in keiner vorteilhaften Lage. Debrecen, im letzten Jahrzehnt zur zweiten Stadt des Landes geworden, verfügt so über beschränktere Möglichkeiten als der Durchschnitt des Städte.

Die Studie erfasst die natürlichen Voraussetzungen für die Freizeit im Umland dieser Stadt, die am Kontakt zweier verschiedener Teile der Ebene liegt. Die Arbeit zeigt zuerst die Dualität der Umgebung der Stadt auf (im Osten ein Raum mit äolischen Sanddünen unter vorherrschender Walbedeckung - der Nyírség ; im Westen ein Lössrücken - Hajdúhát) und setzt dann den Akzent auf die negativen Folgen der Oberflächigen Wasserarmut der Stadt und ihres Umlands. Die Studie widmet sich dann den Versuchen und Projekten zur Bekämpfung der Aridität durch Einleiten des Wassers des Grossen Ostkanals auf Flächen mit sicherem Erholungspotential und analysiert dann den Erholungswert und das Waldsterben des Großen Waldes in Norden der Stadt. Schließlich wird er Verfall der künstlichen Freizeitseen von Erdıspuszta, die in den Dünen von Nyírség angelegt wurden, überprüft und im letzten Teil auf das bescheidene Erholungspotential des Lössrückens im Westen der Stadt hingewiesen.

Zusammenfassend unterstreichen die Autoren, dass der Mangel des für die Entwicklung der Freizeit im umland des Stadt notwendigen Wassers behoben werden könnte, wenn ein Teil des Wassers des Grossen Ostkanals, das derzeit nur für die Stadt bestimmt ist, abgeleitet würde.

INDEX

Keywords : blown sands, Debrecen, drainage, groundwater level, Hungary, loess-region, recreation

Schlüsselwörter : äolische Sande, Debrecen, Dränage, Erholung, Grundwasserspiegel, Lössregion, Ungran

Mots-clés : Debrecen, drainage, Hongrie, loisirs, nappe phréatique, région de lœss, sables éoliens

\section{AUTEURS}

\section{JÓZSEF SZABÓ}

Département de Géographie Physique - Université Lajos Kossuth - Egyetem tér 1, P.O. Box 9 H-4010 Debrecen, HONGRIE

\section{TIMEA KISS}

Département de Géographie Physique - Université Lajos Kossuth - Egyetem tér 1, P.O. Box 9 H-4010 Debrecen, HONGRIE

\section{JÓZSEF LÓKI}

Département de Géographie Physique - Université Lajos Kossuth - Egyetem tér 1, P.O. Box 9 H-4010 Debrecen, HONGRIE 